\title{
NSAID and other analgesic use by endurance runners during training, competition and recovery
}

\author{
J Joslin, MD; J B Lloyd, MD; T Kotlyar, BS; S M Wojcik, PhD \\ Upstate Medical University, Department of Emergency Medicine, Syracuse, New York, USA \\ Corresponding author: J Joslin (joslinj@upstate.edu)
}

\begin{abstract}
Background. An increasing popularity of ultra-endurance events coupled with excessive or inappropriate non-steroidal anti-inflammatory drug (NSAID) use during such events could pose considerable potential risks to runners' health.

Objective. To evaluate the incidence of NSAID and other analgesic use in distance runners during training, competition and recovery. Methods. We performed an observational cross-sectional study at the Desert Race Across the Sand race (Colorado to Utah, USA) in June 2011 and the Empire State Marathon half-marathon, and relay races in Syracuse, NY, October 2011. A total of 27 ultramarathon runners and 46 marathon, half-marathon and marathon relay runners participated in the study. Surveys were distributed to runners during race registration. Self-reported use of common analgesic medications during training, racing and recovery was assessed.

Results. Among all runners at all stages, NSAIDs were the most commonly used analgesic medication. NSAID use by ultramarathon runners compared with all other runners was similar during training $\left(59 \%\right.$ and $63 \%$, respectively; $\left.\chi^{2}=0.008 ; p=0.93\right)$ and recovery $(59 \%$ and $61 \%$, respectively; $\chi^{2}=0.007 ; p=0.93$ ). However, ultramarathon runners were more likely than all other runners to use NSAIDs during the race $\left(70 \%\right.$ and $26 \%$, respectively; $\left.\chi^{2}=11.76 ; p=0.0006\right)$.

Conclusion. Despite undesirable side-effects associated with the use of NSAIDs, there was a high prevalence of use in all runners, particularly during training and recovery. NSAID use during the race was significantly greater in ultramarathon runners. Medical staff at endurance events need to be aware of, and prepared for potential complications related to the high use of NSAIDs in runners. Future efforts should focus on teaching runners about the undesirable effects of medication and emphasising alternatives to pain medication.
\end{abstract}

S Afr J SM 2013;25(4):101-104. DOI:10.7196/SAJSM.495

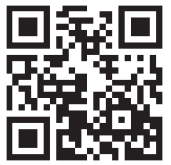

Ultra-endurance sports are increasing rapidly in popularity, with recent exponential growth in the amount of events and athlete participation. ${ }^{[1]}$ These events are characterised by long distances ( $>26.2$ miles) that frequently span wilderness and desert environments over multiple days. While many participants are treated for minor injuries such as blisters, sprains, tendonitis and contusions, ${ }^{[2]}$ some develop more serious ailments like hyponatremia and rhabdomyolysis. ${ }^{[3-6]}$ The extreme nature of these events combined with their rapidly growing participation base creates the potential for increased analgesic use, as compared with marathon and half-marathon participants, seemingly favouring nonsteroidal anti-inflammatory drug (NSAID) consumption.

In all classes of athletes, NSAIDs are one of the most commonly used medications for the treatment of musculoskeletal pain and inflammation. ${ }^{[7,8]}$ In addition to injury treatment, NSAID usage is high as a prophylactic treatment for anticipated pain, ${ }^{[9,10]}$ as well as to enhance the recovery of injuries sustained during an athletic event. ${ }^{[11,12]}$ While NSAIDs are a staple medication for the treatment of certain sports injuries, there is evidence to suggest that many athletes are consuming these medications without adequate knowledge of their intended uses or side-effects. ${ }^{[7,13]}$ Further, there is the potential for athletes to use NSAIDs in dosages exceeding the recommended amount.

The most popular NSAIDs exert their effects through systemic cyclooxygenase (COX) inhibition, thereby reducing the amount of inflammation-causing prostaglandins produced by the body. ${ }^{[14]}$ These effects are not limited to the site of injury and may be detrimental to the healing process of injuries involving bones and tendons. ${ }^{[11]}$ They may also cause potentially dangerous side-effects in the gastrointestinal (GI) tract $^{[15]}$ and kidneys. ${ }^{[16,17]}$ With such a wide catalogue of potential side-effects, it is important to examine the frequency of their use, particularly in ultra-endurance competitors and how they compare with traditional marathon runners ( $\leq 26.2$ miles).

Numerous studies have focused on the incidence of NSAID use during endurance athlete training ${ }^{[5,10,12,16]}$ and competition; ${ }^{[6,18]}$ however, to our knowledge, no study has investigated NSAID and analgesic consumption at multiple timings (training, competition and recovery) at a single event.

The aims of this study were: (i) to evaluate and compare the prevalence of NSAID and other analgesic use during the training, competition and recovery phases of an endurance race in two cohorts (ultra-endurance runners and marathon runners); and (ii) to examine whether participants in both cohorts used NSAIDs in accordance with the recommended dose or in excess thereof.

\section{Methods}

Participants

Runners at two different endurance events, the 2011 Desert Race Across the Sand (RATS) and the 2011 Empire State Marathon (ESM) 
were surveyed to evaluate their patterns of NSAID and analgesic use during training for an event, during a competition, and during recovery following an event. The Desert RATS is a 148-mile ultra-endurance run, performed over six days in individual stages of 9 - 50 miles per stage, across mountainous and desert terrain in Colorado and Utah, USA. In contrast, the ESM is a more typical endurance event with runners participating in a full-marathon, half-marathon, and relays of 6 - 7 miles per runner. The ESM is held in central New York State annually. To the authors' knowledge, no respondent participated in both events.

\section{Data collection}

Data were collected via an anonymous and voluntary survey reviewed and deemed exempt by the institutional internal review board (239319-1). The survey (Table 1) was distributed to runners during race registration at both endurance events.

\section{Statistical analysis}

Using MedCalc (version 11.6.1), data were analysed for differences of proportions between runners' use of analgesia during training, competition and recovery, between those participating in the Desert RATS and ESM races.

\section{Results}

Seventy-three runners participated in the study: 27 ultramarathon runners from the Desert RATS and 46 marathon, halfmarathon and relay runners from the ESM.

NSAIDs were the most commonly reported analgesics used at both races. Fig. 1 shows a comparison of NSAID use delineated by reported habits of using the recommended or greater-than-recommended dose. Runners in the ESM were more likely than those in the Desert RATS race (70\% v. $26 \%$, respectively) to use NSAIDs during a competition $\left(\chi^{2}=11.76 ; p<0.001\right)$. There were no statistical differences in use of NSAIDs between runners participating in the Desert RATS race when compared with those participating in the ESM, during training and recovery.

In both races, acetaminophen was the next most commonly reported analgesic used, followed by topical diclofenac. The percentages of runners utilising these methods of analgesia for both race groups are shown in Table 2 . Aspirin was not used by any participant who

\section{Table 1. Survey distributed to runners during event registration}

1. Which of these do you use either occasionally or regularly during a race to help with pain and inflammation (please select all that apply):

$\square \quad$ Acetaminophen (Tylenol or other)

Do you take more than the recommended dose? $\underline{\text { Yes}} \underline{\underline{\text { No }}}$

$\square \quad$ Ibuprofen (Motrin, Advil or other)

Do you take more than the recommended dose? $\underline{\underline{Y e s}} \underline{\underline{N o}}$

$\square \quad$ Narcotic-based medication (Lortab, Percocet, hydrocodone, oxycodone or other)

Do you take more than the recommended dose? $\underline{\text { Yes/No }}$

$\square \quad$ Aspirin (Bayer or other)

Do you take more than the recommended dose? $\underline{\underline{\text { es }}} \underline{\underline{\mathrm{No}}}$

$\square \quad$ Topical Agent (Voltaren, Diclofenac or other)

Do you take more than the recommended dose? Yes/№

2. Which of these do you use either occasionally or regularly during recovery to help with pain and inflammation (please select all that apply):

$\square \quad$ Acetaminophen (Tylenol or other)

Do you take more than the recommended dose? $\underline{\text { Yes/No }}$

$\square \quad$ Ibuprofen (Motrin, Advil or other)

Do you take more than the recommended dose? $\underline{\text { Yes}} / \underline{\text { No }}$

$\square \quad$ Narcotic-based medication (Lortab, Percocet, hydrocodone, oxycodone or other)

Do you take more than the recommended dose? Yes/ $\underline{\text { No }}$

$\square \quad$ Aspirin (Bayer or other)

Do you take more than the recommended dose? $\underline{\text { Yes/No }}$

$\square \quad$ Topical agent (Voltaren, diclofenac or other)

Do you take more than the recommended dose? $\underline{\text { Yes }} \underline{\underline{\text { No }}}$

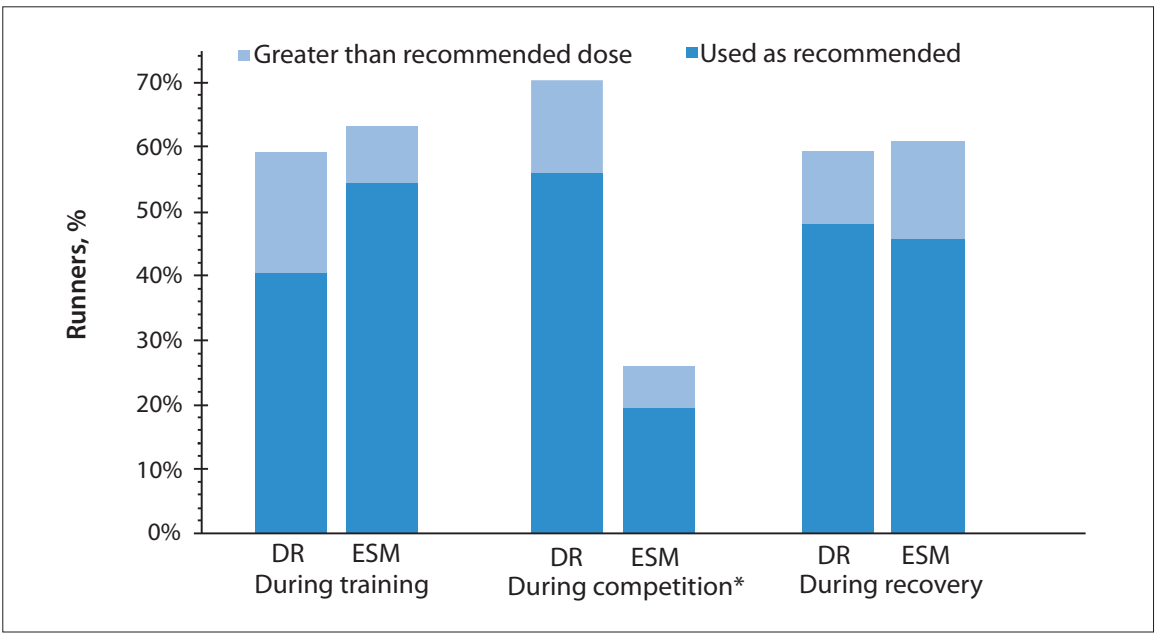

Fig. 1. NSAID use in the Desert RATS (DR) and Empire State Marathon (ESM) runners during training, competition and recovery. The top column represents the fraction of runners that used NSAIDs at dosages higher than the recommended amount. ${ }^{*}$ Significant difference $(\mathrm{p}<0.001)$.

ran in the Desert RATS race, but was used by one participant who ran in the ESM. Narcoticbased medication was reportedly used by only runner of each race group and only during their respective recoveries.

\section{Discussion}

When comparing the prevalence of NSAID use between ultradistance runners and marathon runners, participants reported comparable usage during training $(59 \% \mathrm{v}$. 
Table 2. Acetaminophen and diclofenac use during the Desert RATS and ESM races

\begin{tabular}{|c|c|c|}
\hline & Desert RATS & ESM \\
\hline \multicolumn{3}{|l|}{ During training } \\
\hline Acetaminophen & $26 \%$ & $15 \%$ \\
\hline Topical diclofenac & $19 \%$ & $4 \%$ \\
\hline \multicolumn{3}{|l|}{ During competition } \\
\hline Acetaminophen & $11 \%$ & $11 \%$ \\
\hline Topical diclofenac & $7 \%$ & $2 \%$ \\
\hline \multicolumn{3}{|l|}{ During recovery } \\
\hline Acetaminophen & $11 \%$ & $17 \%$ \\
\hline Topical diclofenac & $4 \%$ & $4 \%$ \\
\hline
\end{tabular}

$63 \%$ ) and during recovery (59\% v. $61 \%)$, but with a large discrepancy of usage during a race ( $70 \%$ v. $26 \%$ ). These results do not suggest that more ultradistance runners use NSAIDs during training or recovery than traditional marathon runners. The similarity in training and recovery NSAID use is expected, given that both types of events would require relatively homogenous preparation and recuperation routines. Additionally, this similarity could be used as a basis for comparison between the two participant types, highlighting the major difference between the two cohorts: the elevated number of NSAID users in the ultradistance event. This difference could be rationalised by the increased duration and intensity of ultradistance events, as compared with marathons, increasing the probability that an athlete may experience pain and turn to NSAIDs.

Prophylactic use of NSAIDs is common in endurance events ${ }^{[5,10,12,16]}$ with 59\% of Ironman Brazil triathletes reporting consumption in the three months preceding the event, ${ }^{[7]}$ a number comparable with this study where $59 \%$ of ultramarathon runners and $63 \%$ of marathon runners reported using NSAIDs during training. However, in addition to gastrointestinal and renal side-effects, prophylactic NSAID use may impede collagen synthesis and reduce tissue adaptation, predisposing the athlete to future injury. ${ }^{[16]}$ Additionally, many believe that the analgesic effect of NSAIDs can mask the pain of an injury and thus prevent the athlete from resting enough to allow for proper healing. ${ }^{[17]}$

High levels of NSAID use during endurance events are fairly common, with self-reported usage ranging from $30 \%$ at the Ironman New Zealand Ultradistance Triathlon ${ }^{[18]}$ to $61 \%$ at the 2002 Boston Marathon. ${ }^{[6]}$ A study of 500 runners in two major North American ultramarathons showed that $60.5 \%$ of race finishers used NSAIDs during the race and only $28.7 \%$ of finishers did not use any medication at all, ${ }^{[1]}$ and our survey reaffirms this difference. However, the significantly higher rate of NSAID consumption during a race by the ultradistance cohort $v$. the marathon cohort is concerning, and may put the former group at an elevated risk for adverse events.

Two major risks of NSAID use during an ultra-endurance race are the development of renal complications and exercise-associated hyponatraemia (EAH). NSAIDs reduce prostaglandin synthesis in the kidney, decreasing the glomerular filtration rate and afferent renal blood flow, which may already be lowered from exercise by as much as $50 \%{ }^{[15-17]}$ This combination can impair the athlete's free water clearance, increasing the risk for developing renal disease and/ or EAH. ${ }^{[1,5,7,8,17-21]}$ The National Kidney Foundation estimates that $10 \%$ of kidney failure is associated with NSAID overuse. ${ }^{[15]}$ While there are many studies that demonstrate a link between NSAID use and increased renal complications in athletes, ${ }^{[1,17-20]}$ other studies show that no such correlation exists. ${ }^{[5]}$ However, there is consensus that NSAIDs are a clear risk factor for renal disease and EAH, and it is recommended that these drugs are avoided by endurance athletes, particularly in the wilderness ultramarathon setting. ${ }^{[21,22]}$

An additional risk factor for NSAID-related adverse events is taking more than the recommended dose. This study did not find any significant difference between NSAID-using ultradistance runners and marathon runners with regards to surpassing the recommended dose in the period during training $(31 \% \mathrm{v}$. $14 \%)$, a race $(21 \% \mathrm{v}$. $25 \%)$ and recovery (19\% v. $25 \%)$, respectively. These results do not suggest that ultra-distance runners are at an increased risk from taking NSAIDs above the recommended dose, but only as compared with marathon runners. A potential future study could look to determine and compare the amount by which each cohort exceeds the recommended dose.

As attested to by its common use reported in this survey, acetaminophen may be a better alternative to NSAIDs for minor pain reduction, due to its milder effect on the GI tract, kidneys, and tissue regeneration. ${ }^{[15,20]}$ Acetaminophen's main adverse effects come from overdose-induced hepatic toxicity, and some evidence suggests that its pain-relieving properties may be comparable to those of NSAIDs. ${ }^{[14]}$ Topical agents (such as diclofenac) are another group of analgesics used by athletes, albeit in reduced numbers (19\% of ultramarathon runners during training in this study). The subcutaneous delivery to a localised site of injury makes these agents effective at treating minor acute musculoskeletal pain, ${ }^{[12,14,15,20,23]}$ while avoiding many of the severe adverse reactions that NSAIDs carry. ${ }^{[1,20]}$

Encouragingly, an unpublished survey by one co-author found that $59 \%$ of athletes surveyed would modify the frequency of their NSAID use if they were better educated on their potentially harmful effects. This represents an opportunity for medical providers involved in this sport's industry to improve the health and safety of endurance race participants. One particular opportunity for education, at least popular in ultramarathon events, is the pre-race medical briefing, where medical directors and race staff have the ability to provide evidencebased guidelines to runners before the event commences.

\section{Conclusion}

Despite the well-documented, undesirable side-effects associated with NSAID use, there was a high incidence of use in our cohort of runners, particularly during a race in the ultramarathon group. More effort should be made to inform runners about the potential hazards of NSAID misuse and to offer alternatives to oral NSAIDs for minor pain relief. If necessary, NSAID use should be limited to a short-term regimen, taken after the injury, and for the intended purposes only.

Acknowledgements. The authors acknowledge Nathaniel Herr for assistance with data collection and William Grant for assistance with manuscript preparation. 


\section{References}

1. Hoffman MD, Fogard K. Factors related to successful completion of a 161-km ultramarathon. Int J Sports Physiol Perform 2011;6(1):25-37.

2. McLaughlin KA, Townes DA, Wedmore IS, et al. Pattern of injury and illness during expedition-length adventure races. Wilderness Environ Med 2006;17(3):158-161. [http:// dx.doi.org/10.1580/PR29-05]

3. Hew TD, Chorley JN, Cianca JC, et al. The incidence, risk factors, and clinical manifestations of hyponatremia in marathon runners. Clin J Sport Med 2003;13(1):41-47.

4. Bruso JR, Hoffman MD, Rogers IR, et al. Rhabdomyolysis and hyponatremia: A cluster of five cases at the 161-km 2009 Western States Endurance Run. Wilderness Environ Med 2010;21(4):303-308. [http://dx.doi.org/10.1016/j.wem.2010.06.012]

5. Page AJ, Reid SA, Speedy DB, et al. Exercise-associated hyponatremia, renal function, and nonsteroidalantiinflammatory drug use in an ultraendurance mountain run. Clin J Sport Med Off J Can Acad Sport Med 2007;17(1):43-48. [http://dx.doi.org/10.1097/ JSM.0b013e31802b5be9]

6. Almond CSD, Shin AY, Fortescue EB, et al. Hyponatremia among runners in the Boston Marathon. N Engl J Med 2005;352(15):1550-1556. [http://dx.doi.org/10.1056/ NEJMoa043901]

7. Gorski T, Cadore EL, Pinto SS, et al. Use of NSAIDs in triathletes: Prevalence, level of awareness and reasons for use. Br J Sports Med 2011;45(2):85-90. [http://dx.doi. org/10.1136/bjsm.2009.062166]

8. Ciocca M. Medication and supplement use by athletes. Clin Sports Med 2005;24(3):719738. [http://dx.doi.org/10.1016/j.csm.2005.03.005]

9. Warden SJ. Prophylactic misuse and recommended use of non-steroidal antiinflammatory drugs by athletes. Br J Sports Med 2009;43(8):548-549. [http://dx.doi. org/10.1136/bjsm.2008.056697]

10. Tscholl P, Alonso JM, Dollé G, et al. The use of drugs and nutritional supplements in top-level track and field athletes. Am J Sports Med 2010;38(1):133-140. [http://dx.doi. org/10.1177/0363546509344071]

11. Mehallo CJ, Drezner JA, Bytomski JR. Practical management: Nonsteroidal antiinflammatory drug (NSAID) use in athletic injuries. Clin J Sport Med 2006;16(2):170174.

12. Ziltener J-L, Leal S, Fournier P-E. Non-steroidal anti-inflammatory drugs for athletes:
An update. Ann Phys Rehabil Med 2010;53(4):278-282. [http://dx.doi.org/10.1016/j. rehab.2010.03.001]

13. Warner DC, Schnepf G, Barrett MS, et al. Prevalence, attitudes, and behaviors related to the use of nonsteroidal anti-inflammatory drugs (NSAIDs) in student athletes. J Adolesc Heal 2002;30(3):150-153. [http://dx.doi.org/10.1016/S1054-139X(01)00325-1]

14. Feucht CL, Patel DR. Analgesics and anti-inflammatory medications in sports: Use and abuse. Pediatr Clin North Am 2010;57(3):751-774. [http://dx.doi.org/10.1016/j. pcl.2010.02.004]

15. Lilly KF. Athletes, NSAID, coxibs, and the gastrointestinal tract. Curr Sports Med Rep 2010;9(2):103-105

16. Warden SJ. Prophylactic use of NSAIDs by athletes: A risk/benefit assessment. Phys Sportsmed 2010;38(1):132-138. [http://dx.doi.org/10.3810/psm.2010.04.1770]

17. Baker J, Cotter JD, Gerrard DF, et al. Effects of indomethacin and celecoxib on renal function in athletes. Med Sci Sports Exerc 2005;37(5):712-717. [http://dx.doi. org/10.1249/01.MSS.0000162700.66214.CE]

18. Wharam PC, Speedy DB, Noakes TD, et al. NSAID use increases the risk of developing hyponatremia during an Ironman triathlon. Med Sci Sports Exerc 2006;38(4):618-622. [http://dx.doi.org/10.1249/01.mss.0000210209.40694.09]

19. Davis DP, Videen JS, Marino A, et al. Exercise-associated hyponatremia in marathon runners: A two-year experience. J Emerg Med 2001;21(1):47-57. [http://dx.doi. org/10.1016/S0736-4679(01)00320-1]

20. Alaranta A, Alaranta H, Helenius I. Use of prescription drugs in athletes. Sports Med 2008;38(6):449-463. [http://dx.doi.org/10.2165/00007256-200838060-00002]

21. Rogers IR, Hew-Butler T. Exercise-associated hyponatremia: Overzealous fluid consumption. Wilderness Environ Med 2009;20(2):139-143. [http://dx.doi. org/10.1580/08-WEME-CON-231R2.1]

22. Lipman GS, Chan G, Logan S, et al. A prospective cohort study of acute kidney injury in multi-day ultramarathon runners. Wilderness Environ Med 2011;22(4):358. [http:// dx.doi.org/10.1016/j.wem.2011.08.019]

23. Galer BS, Rowbotham M, Perander J, et al. Topical diclofenac patch relieves minor sports injury pain: Results of a multicenter controlled clinical trial. J Pain Symptom Manage 2000;19(4):287-294. [http://dx.doi.org/10.1016/S0885-3924(00)00125-1] 